\title{
Transfer of lithium foil under inert conditions using CleanConnect inert gas transfer system
}

Krishna Kanth Neelisetty ${ }^{1}$, Jakub Stetina ${ }^{1}$, Jindřich Vondruška ${ }^{2}$, Milos Trenz ${ }^{3}$, Tomas Kazda ${ }^{4}$, Michal Hrouzek $^{1}$ and Petr Wandrol ${ }^{5}$

${ }^{1}$ Thermofisher Scientific, Brno, Jihomoravsky kraj, Czech Republic, ${ }^{2}$ Thermofisher Scientific, Jihomoravsky kraj, Czech Republic, ${ }^{3}$ Thermofisher Scientific, Brno, Czech Republic, ${ }^{4}$ Brno University of Technology, Brno, Jihomoravsky kraj, Czech Republic, ${ }^{5}$ Thermo Fisher Scientific, Brno, Jihomoravsky kraj, Czech Republic

Recent advances in microscopy are opening exciting directions in nanomaterials research. The possibilities to combine various microscopy methods to study applied materials e.g. batteries, volatile memories, sensors, etc. are increasing and in parallel also the challenges towards characterization of these materials [1]. The materials used for certain applications like batteries are oxygen/air sensitive and the characterization also requires oxygen-free sample handling tools during the transfer process for correlative microscopy. In most of the cases, oxygen/air sensitive samples are prepared and stored in glove box and are transferred to an electron microscope for further microscopic characterization studies. The transfer process from the glove box to these tools takes couple of minutes and the samples can be oxidized during the transfer in atmosphere.

CleanConnect is an inert gas transfer system developed to address this challenge where oxygen/air sensitive samples are successfully transferred between glovebox and scanning electron microscope (SEM) under inert conditions (Ar or vacuum) [2]. The following figure 1a show the CleanConnect system. It consists of three main parts, interface (installed on SEM chamber and/or glovebox), shuttle and transfer rod. Shuttle can be detached from the interface and transfer rod. It can be inserted into glove box through load lock or connected via interface on glovebox. The sample is stored inside shuttle where vacuum or high pressure $\mathrm{Ar} / \mathrm{N}$ is maintained.

The following Figure $1 \mathrm{~b}$ shows the time vs relative pressure plot where over pressure of $\sim 7$ mbar and 20 mbar is maintained for 1 hour in the CleanConnect shuttle. These measurements show that there is not considerable leak in the shuttle. The initial drop (enclosed images) between 0.1-0.2 \& 0.2-0.3 minutes is caused when the shuttle valve is closed. Figure $2 \mathrm{~b}$ shows the stability of the CleanConnect shuttle under vacuum.

In order to confirm the measurements of the pressure stability, pure lithium (highly reactive alkali metal) was used as a test sample to look for the possible reaction to oxygen/air during transfer under inert conditions. The sample was placed on a stub in the glovebox. CleanConnect was used for transfer process. The transfer time for this measurement was $\sim 1$ hour (due to long distance between tools). Figure 2a shows the backscattered electron (BSE) image of the Li surface after transfer from glovebox to SEM. Figure 2b shows the same area of $\mathrm{Li}$ after another 1 hour of storing the sample in CleanConnect shuttle under Ar atmosphere. These two images show that the there are no considerable changes in the surface morphology. And figure 2c (SE image) is after the sample is stored in atmosphere for $~ 30$ min where we could see change in surface morphology due to reaction with air. 
To conclude, the CleanConnect system demonstrated a perfect solution for efficient transfer of highly reactive samples such as lithium under low pressures ( 2-3 mbar) of Ar. It can be easily used to transfer samples from standard laboratory equipment such as a glove box with/without the need of additional modifications to these devices. However, it is not suitable for long-term storage of sensitive samples.

a

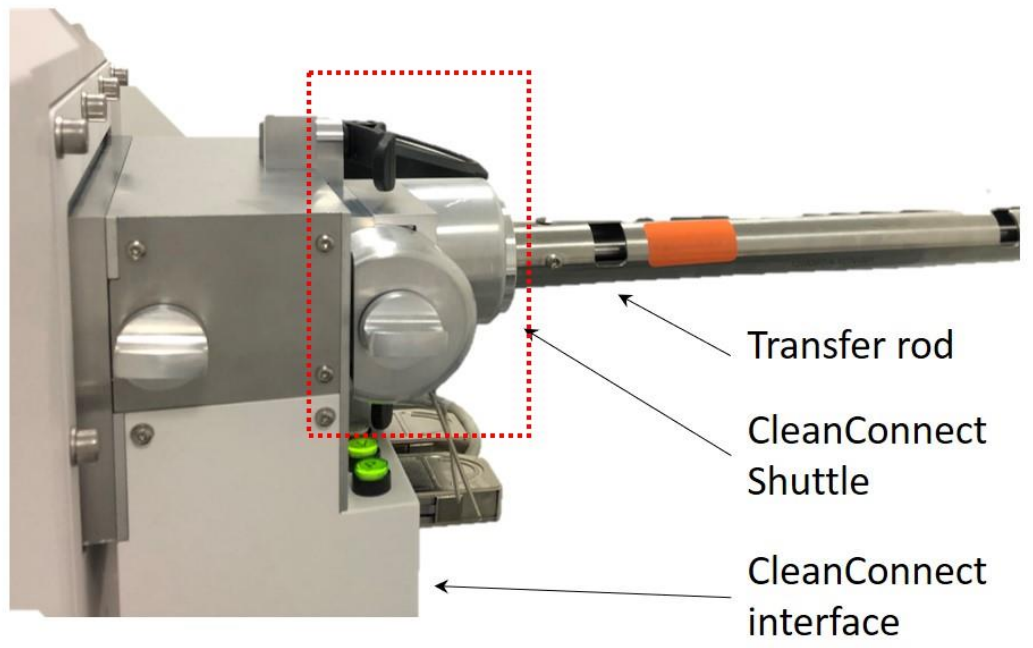

\section{b}
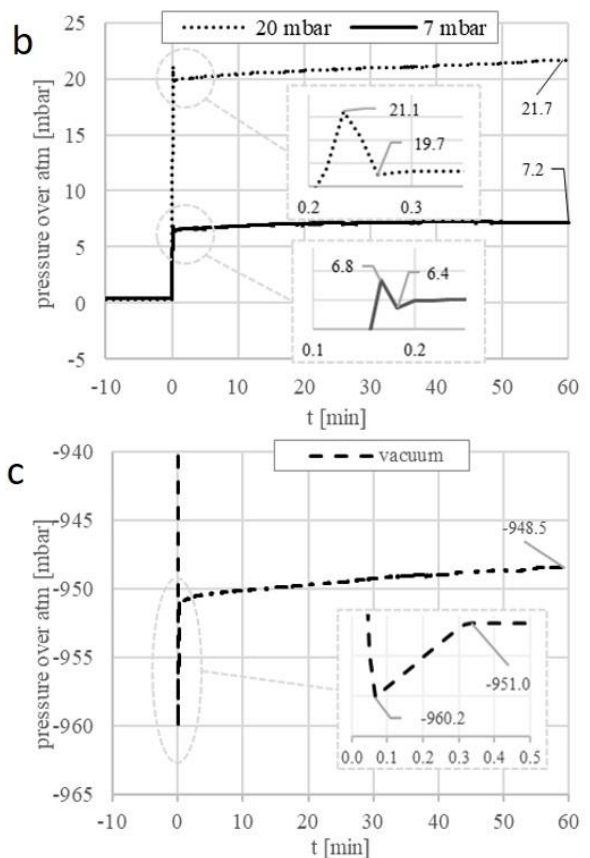

Figure 1. a. Clean connect Inert gas transfer system; b. Over pressure vs time plot shows the stability of clean connect for 1 hour; c. Vacuum vs time plot of the CC shows the stability for 1 hour
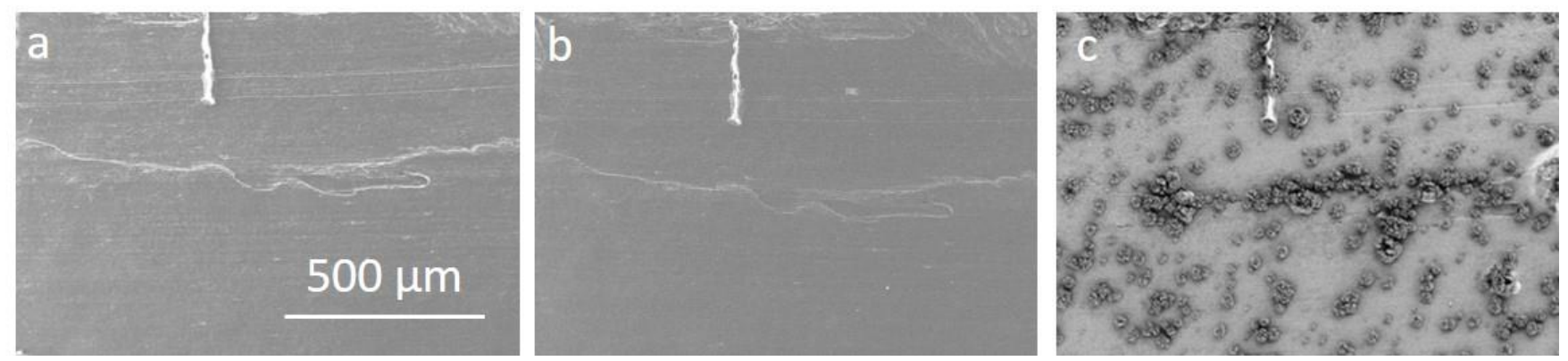

Figure 2. a. BSE images of Li a. as transferred (1 hour) from the glove box; b. stored in the Clean connect under Ar atmosphere for 1 hour; c. SE image of same area in atmosphere for $30 \mathrm{~min}$

\section{References}

[1] Liu, Y., et al, (2019), "Challenges and opportunities towards fast-charging battery materials" Nat Energy 4, 540-550. https://doi.org/10.1038/s41560-019-0405-3

[2] Sebastian Tacke, et al, (2016), "A Versatile High-Vacuum Cryo-transfer System for Cryo-microscopy and Analytics” Biophysical Journal, Volume 110, Issue 4, 758-765, https://doi.org/10.1016/j.bpj.2016.01.024 